\title{
Mirizzi syndrome with an unusual aberrant hepatic duct fistula: a case report
}

This article was published in the following Dove Press journal:

International Medical Case Reports Journal

4 July 2016

Number of times this article has been viewed

\author{
Meng Wang' \\ Yufei Xing ${ }^{2}$ \\ Quangen Gao' \\ Zhiqiang Lv' $^{\prime}$ \\ Jianmao Yuan' \\ 'Department of General Surgery, The \\ First People's Hospital of Wujiang, \\ Affiliated Wujiang Hospital of Nantong \\ University, ${ }^{2}$ Digestive Clinical Center, \\ The Second Affiliated Hospital of \\ Soochow University, Suzhou, Jiangsu, \\ People's Republic of China
}

\begin{abstract}
Mirizzi syndrome (MS) is a rare complication of chronic cholelithiasis, which is always caused by a calculus in the cystic duct or neck of the gallbladder, resulting in mechanical compression of common bile duct and the gallbladder. It is clinically characterized by abdominal pain, fever, as well as obstructive jaundice. During cholecystectomy, MS is seen as a dangerous adherent and inflammatory tissue in the area of Calot's triangle. In the general population, aberrant right posterior hepatic duct, one of the causes of bile duct injury during duct surgery, is present in $4.8 \%-8.4 \%$ of people. Herein we report a rare case of a 76 -year-old female patient, with hepatolithiasis of right posterior lobe and cholecysto-aberrant right posterior hepatic duct fistula. This is a special type of MS; however, interestingly, she did not have any symptoms, and the disease was found by physical examination incidentally. This case highlights another situation, namely, there may be difficulty in diagnosing MS and dissecting for operation. Therefore, to avoid the complication associated with this special situation, the surgeons need to diagnose carefully and adopt an optimal treatment strategy.
\end{abstract}

Keywords: Mirizzi syndrome, aberrant hepatic duct, hepatolithiasis, anatomical hepatectomy

\section{Background}

Mirizzi syndrome (MS), an unusual complication of gallstone disease, was first described by the Argentinean surgeon Pablo Mirizzi based on cholangiography in $1948 .{ }^{1}$ It is rare and occurs in only $0.7 \%-1.4 \%$ of gallstone patients; the typical signs are intermittent or constant jaundice, fever, and right upper quadrant abdominal pain. Although these symptoms are found in approximately $80 \%$ of cases, there is no specific clinical picture of MS. As a result, it is difficult to identify it preoperatively. ${ }^{2} \mathrm{MS}$ can be confirmed only when the typical cholangiography revealed a smooth structure of common bile duct (CBD). Although some advanced technologies, such as magnetic resonance cholangiopancreatography (MRCP), multidetector computed tomography (MDCT), and ultrasound (US) can assist in preoperative diagnosis, MS with fistula is mainly diagnosed during operation. The CBD or hepatic duct may be involuntarily injured during duct operation, and the former is easily injured by technical failures, causing an aberrant duct. Therefore, more appropriate surgical procedures need to be adopted for MS with an aberrant duct fistula. We herein reported a case of MS with an unusual type fistula that was successfully treated with the combined approach of partial hepatectomy, cholecystectomy, and T-tube drainage.
Correspondence: Meng Wang Department of General Surgery, The First People's Hospital of Wujiang, Affiliated Wujiang Hospital of Nantong University, 169 Gongyuan Road, Suzhou 215200, Jiangsu, People's Republic of China

Tel/fax +8651263024562

Email wangmeng2004-2008@163.com 


\section{Case presentation}

The patient, a 76-year-old Asian female, was detected with liver gallstones (lithiasis) by ultrasonic diagnosis during a health examination and was admitted to our department. At that time, she did not show fatigue, jaundice, intermittent high fever, and right upper quadrant abdominal pain. There was no significant medical background and family history. Moreover, according to the abdominal findings via physical examination, no icteric scleras and right upper quadrant abdominal tenderness in deep palpation were found, nor were the rebound tenderness or abdominal muscle rigidity and palpable masses found. The following were the testing indexes:

1. Blood tests showed ALT: $313 \mathrm{U} / \mathrm{L}$ (normal: $0-40 \mathrm{U} / \mathrm{L}$ ), AST: 443 U/L (normal: 0-40 U/L), ALP: 67 U/L (normal: $<106 \mathrm{U} / \mathrm{L}$ ), and $\gamma$-GGT: $93 \mathrm{U} / \mathrm{L}$ (normal: <50 U/L) with normal bilirubin levels: $20 \mu \mathrm{mol} / \mathrm{L}$ (normal values for total bilirubin: $0-25 \mu \mathrm{mol} / \mathrm{L}$ ).

2. Serologic tests for hepatitis $\mathrm{B}$ and $\mathrm{C}$ were all negative. Tumor markers including $\alpha$-fetoprotein, carcinoembryonic antigen, and carbohydrate antigen 19-9 were within normal limits.

3. US examination showed cholelithiasis with a distended gallbladder, multiple calculi within its lumen, and moderate intrahepatic biliary dilatation.

4. Abdominal enhanced multidetector computed tomography scan revealed an atrophic right posterior liver and a dilated hepatic duct and right posterior sectional duct with multiple stones inside.

5. MRCP indicated the presence of a sinus tract between the gallbladder fossa and right posterior hepatic duct. Cholecystobiliary and aberrant right posterior hepatic duct fistula were also observed (Figure 1). Computed tomographic (CT) scans showed multiple dilated bile ducts in the right posterior segment with stones (Figure 2).

Although the patient was suffering from severe lithiasis, she did not show any symptoms. On the basis of the aforementioned data, the initial diagnosis before operation was MS complicated with intrahepatic lithiasis associated with aberrant right posterior hepatic duct fistula. Surgery remained the exclusive choice of curative therapy, and the definitive diagnosis was confirmed intraoperatively. An exploratory laparotomy through a right subcostal incision was performed. During cholecystectomy, two severe anatomic alterations were observed. The first was an impact stone found in the cystic duct, which firmly adhered to the aberrant right posterior hepatic duct and the cholecys- tobiliary fistula, and involved less than one-third of the circumference of the duct. The other was a wide range of tissue adhesion, which was associated with elongated cystic duct, parallel to the CBD. Right posterior lobe was resected. Multiple cystic dilations of the intrahepatic biliary tree and black pigment stones adherent to the bile duct wall were observed in the resected specimen (Figure 3 ). The CBD was lavaged, and the defect of the CBD was sutured in a one-layer manner. Finally, a T-tube was inserted. The postoperative course was uneventful and the patient was discharged a week after the operation. Routine follow-up was continued in the hepatobiliary clinic.

\section{Discussion}

MS, a rare complication ( $1 \%)$ of chronic cholecystitis and cholelithiasis, was first described in 1923 by Hans Kehr and was characterized by impaction of stones in the cystic duct or neck of the gallbladder, resulting in mechanical compression or erosion of the CBD. ${ }^{1}$ As there is no specific clinical or laboratory finding for the syndrome, MS was always characterized with jaundice, abdominal pain, and alterations during the serum tests of hepatic function. However, these symptoms of MS are seen in $\sim 80 \%$ of cases. ${ }^{2}$ The patient we reported here had no significant clinical picture. Even with the availability of modern imaging techniques, majority of cases are not identified preoperatively.

McSherry et $\mathrm{al}^{3}$ classified MS into two types based on endoscopic retrograde cholangiopancreatography (ERCP) findings: Type I MS when there is extrinsic compression of the common hepatic duct and Type II MS when the gallstones erode the common hepatic duct wall, resulting in a cholecystocholedochal fistula. The most recent and important classification was made by Nagakawa et al, ${ }^{4}$ while probably the best one was done by Csendes et al. ${ }^{5}$ According to the classification of Csendes et $\mathrm{al}^{5}$, MS is divided in four types depending on the size of the destruction of the CBD. ${ }^{6} \mathrm{We}$ consider this case as a special kind of MS where intrahepatic stones complicated patient's condition. In general, a preoperative diagnosis is crucial for us to choose the appropriate surgical approach.

US was always utilized as a primary investigation in the treatment of patients with abdominal pain and jaundice. MS was confirmed via the evidence of dilated intrahepatic ducts and common hepatic duct by observing the narrowness of the compressed external bile duct that caused by the calculus. ${ }^{7}$ It was regarded as a criterion used to diagnose MS that cholangiographic evidence of stone affected compression of the bile duct in the gallbladder neck or cystic duct, or the presence 

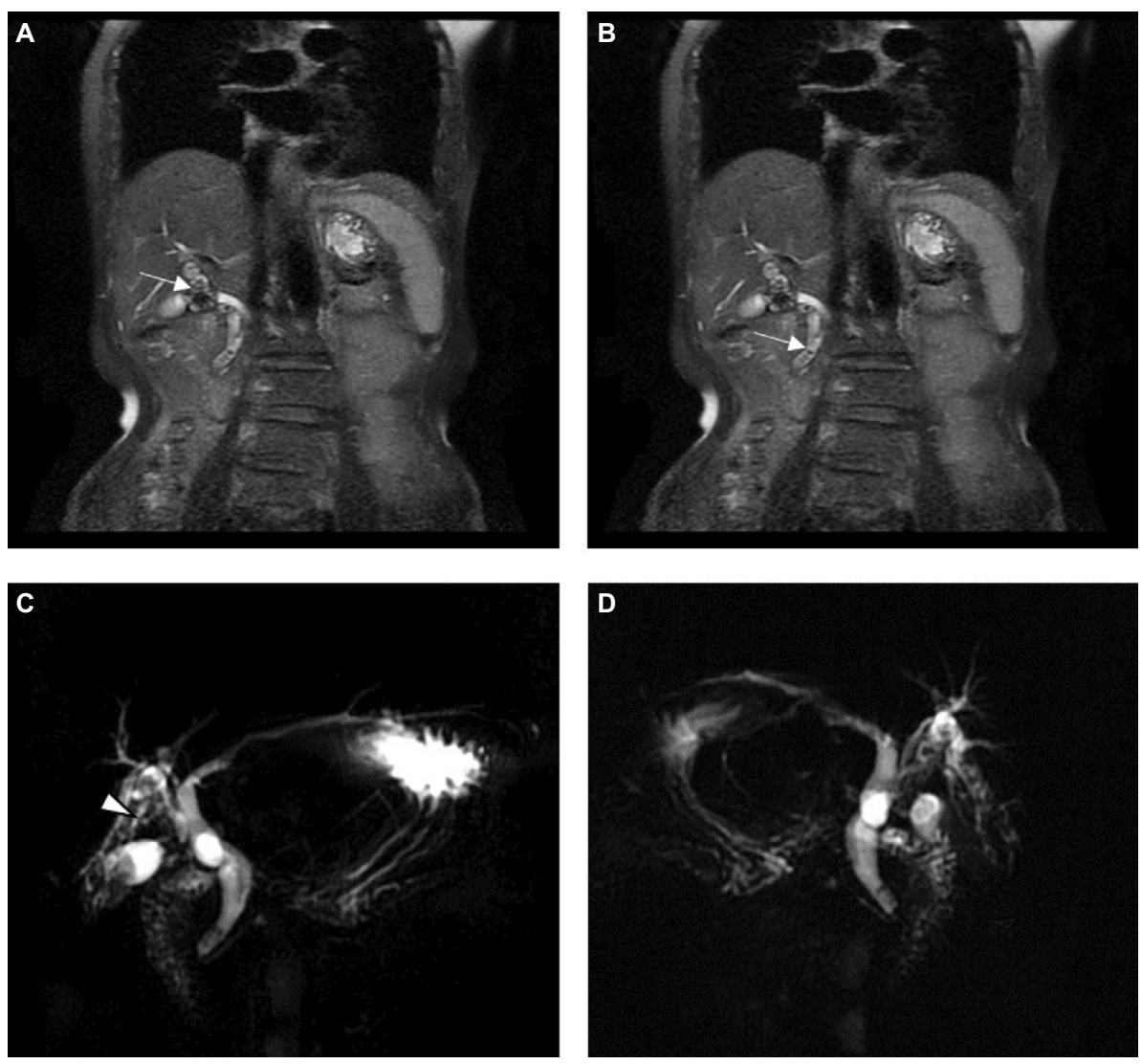

Figure I Preoperative magnetic retrograde cholangiopancreatography demonstrating a gallstone in the cystic duct (A, arrow), stones in the CBD (B, arrow), aberrant right posterior duct (C, arrowhead), and proximal dilatation of the biliary tree (A-D). Abbreviation: $\mathrm{CBD}$, common bile duct.

of a cholecystcholedochal fistulam, which were detected by CT, MRCP, and ERCP. In addition, direct cholangiography is the gold standard for locating subtle abnormalities within the bile ducts and detecting small calculi. ${ }^{8,9}$ Nevertheless, the patient refused ERCP, even though it remains the gold standard for preoperative diagnoses of MS. A recent study demonstrated that MRCP, a noninvasive alternation to ERCP, is a useful preoperative diagnostic modality for MS with a higher accuracy and better interobserver agreement when combined with $\mathrm{CT} .{ }^{10}$ In this case, MRCP demonstrated a possibility of an already existing abnormal fistula between the gallbladder and the common hepatic duct. Noninvasive imaging modalities such as ultrasonography, CT, and magnetic resonance imaging can also accurately depict the presence of intrahepatic stones. The patient was diagnosed with intrahepatic lithiasis that caused liver fibrosis, atrophy, and fibrotic stricture of right posterior hepatic duct and her second/third branches.

The incidence of hepatolithiasis has been reported to be $20 \%-31 \%$ in the patients with gallstone disease, and it is a prevalent disease in East Asia. Compared to nonoperative treatment, hepatic resection is a preferable choice for unilateral stones treatment. ${ }^{11}$ It was reported that surgical therapy is a safe and effective management for hepatolithiasis. However, MS hinders the complex operative procedure further, by making it much more difficult on the condition of dangerous anatomical alteration in the area of Calot's triangle.

As the principle of which is to remove the stones as well as the biliary stricture and to reduce the risk of recurrent stones, hepatectomy seems to be the optimal treatment for hepatolithiasis among selected patients. ${ }^{12-14}$ Tabrizian et al's ${ }^{15}$ investigation showed that hepatic resection is a safe and definitive treatment option in the control of primary hepatolithiasis, and it could achieve excellent short- and longterm effect. Furthermore, according to Jiang et al's ${ }^{11}$ study, anatomical hepatectomy can produce better clinical outcome than limited hepatectomy about regional hepatolithiasis. In our study, hepatolithiasis was limited to a right posterior lobe (segment) irrespective of lobular atrophy or fibrosis. By receiving anatomical hepatectomy, she may get a radical cure so as to minimize the action on hepatic function. During operation, MS was confirmed only after the extraction of large gallstone that compacted on gallbladder neck and right posterior hepatic duct, and under this circumstance, 

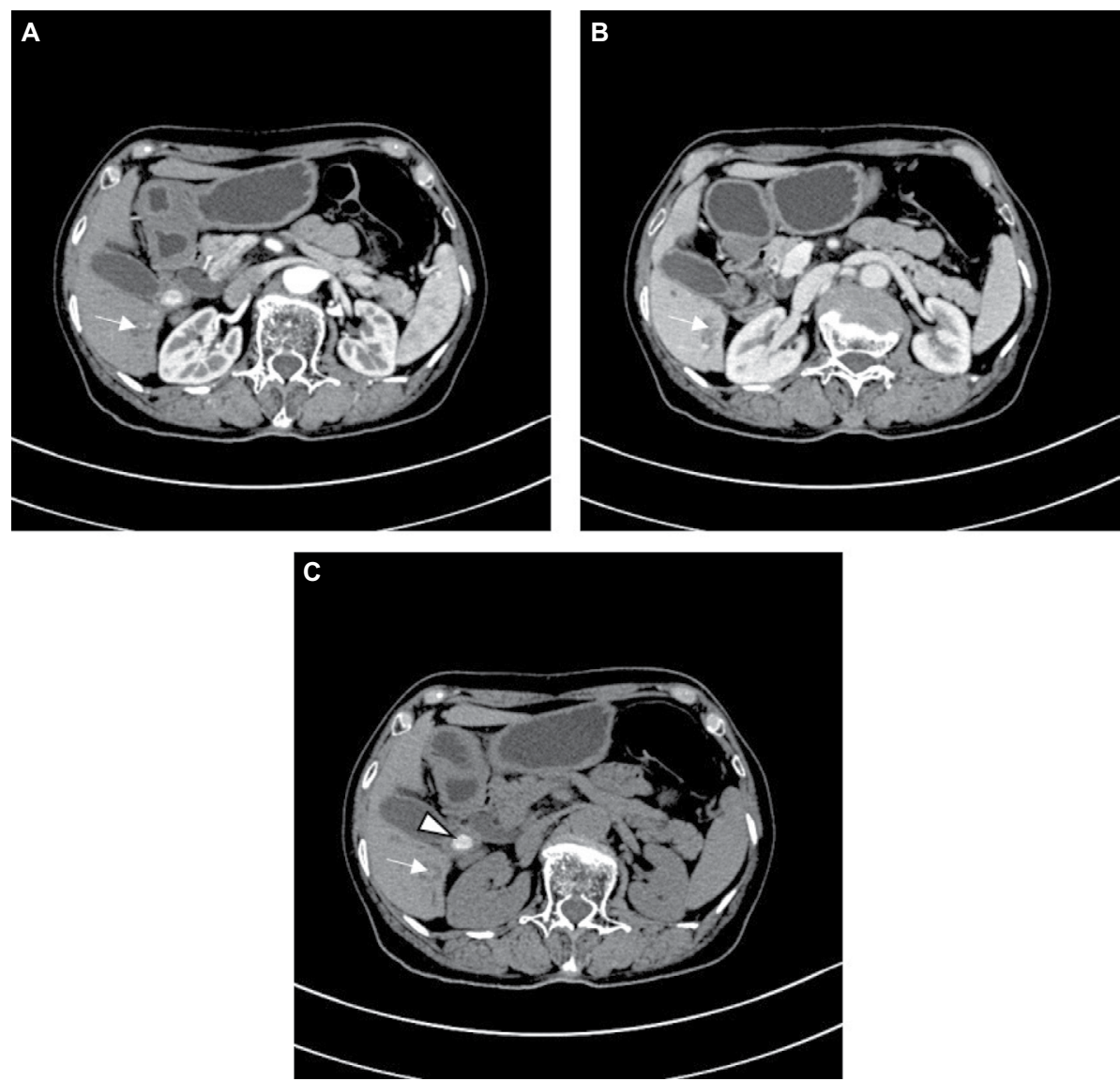

Figure 2 Preoperative computed tomographic scans showing multiple dilated bile ducts in the right posterior segment with stones (A-C, arrow) and a gallstone in the cystic duct (C, arrowhead).

Notes: (A) hepatic arterial phase; (B) portovenous phase, and (C) plain scan.

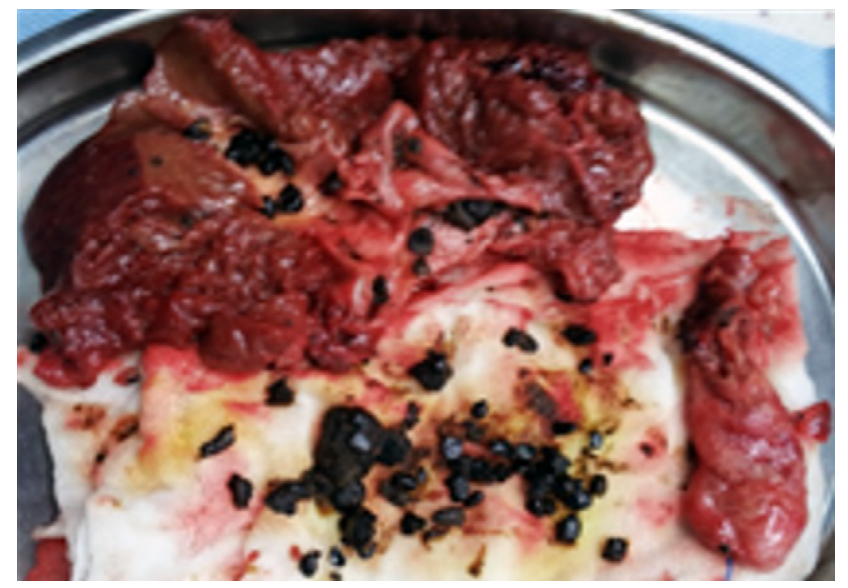

Figure 3 Hepatectomy specimen with pigmented stones within irregularly dilated ducts.

the cystic duct usually ran parallel to the CBD $(10 \%$ of cholangiograms). ${ }^{16}$

MS exhibited a dangerous and unexpected variation in Calots' triangle. Thus, preoperative recognition of this variation is significantly important to avoid inadvertent ligation or severance of the bile duct. The fistula's excision and repair of the hepatic flexure with primary closure was finally performed without any further postoperative complications. A T-tube was routinely inserted after hepatectomy for postoperative cholangiography and choledochoscopy via the T-tube route.

\section{Consent}

This protocol was approved by the Institutional Review Board of The First People's Hospital of Wujiang. Written informed consent was obtained from the patient for the publication of this case report and any accompanying images.

\section{Conclusion}

Preoperative evaluation of patient's condition, adequate recognition of variation, right approach, and minimal surgical maneuvers at the time of the operation were important key points for successful treatment of these clinical entities.

\section{Acknowledgment}

We thank Genhai Shen who provided several kind suggestions in preoperative evaluation. 


\section{Disclosure}

The authors report no conflicts of interest in this work.

\section{References}

1. Colovic R. Was Mirizzi really the first person to recognize Mirizzi syndrome? Int J Surg. 2013;11:1022-1023.

2. Lampropoulos P, Paschalidis N, Marinis A, Rizos S. Mirizzi syndrome type Va: a rare coexistence of double cholecysto-biliary and cholecystoenteric fistulae. World J Radiol. 2010;2(10):410-413.

3. McSherry CK, Ferstenberg H, Calhoun WF, Lahman E, Virshup M The natural history of diagnosed gallstone disease in symptomatic and asymptomatic patients. Ann Surg. 1985;202(1):59-63.

4. Nagakawa T, Ohta M, Kayahara K, et al. A new classification of Mirizzi syndrome from diagnostic and therapeutic viewpoints. Hepatogastroenterology. 1997;44:63-67.

5. Csendes A, Diaz JC, Burdiles P, Maluenda F, Nava O. Mirizzi syndrome and cholecystobiliary fistula: a unifying classification. Br J Surg. 1989; 76(11):1139-1143.

6. Waisberg J, Corona A, de Abreu IW, Farah JF, Lupinacci RA, Goffi FS. Benign obstruction of the common hepatic duct (Mirizzi syndrome): diagnosis and operative management. Arq Gastroenterol. 2005;42(1):13-18

7. Dewbury KC. The features of the Mirizzi syndrome on ultrasound examination. Br J Radiol. 1979;52(624):990-992.
8. Wang WK, Yeh CN, Jan YY. Successful laparoscopic management for cholecystoenteric fistula. World J Gastroenterol. 2006;12(5):772-775.

9. Gomez D, Rahman SH, Toogood GJ, et al. Mirizzi's syndrome results from a large western experience. HPB (Oxford). 2006;8(6): 474-479.

10. Yun EJ, Choi CS, Yoon DY, et al. Combination of magnetic resonance cholangiopancreatography and computed tomography for preoperative diagnosis of the Mirizzi syndrome. J Comput Assist Tomogr. 2009;33(4): 636-640.

11. Jiang $\mathrm{H}, \mathrm{Wu} \mathrm{H}, \mathrm{Xu} \mathrm{YL}$, Wang JZ, Zeng Y. An appraisal of anatomical and limited hepatectomy for regional hepatolithiasis. HPB Surg. 2010; 2010:791625.

12. Fan ST, Lai EC, Wong J. Hepatic resection for hepatolithiasis. Arch Surg. 1993;128(9):1070-1074.

13. Chen DW, Tung-Ping PR, Liu CL, Fan ST, Wong J. Immediate and long-term outcomes of hepatectomy for hepatolithiasis. Surgery. 2004; 135(4):386-393.

14. Uchiyama K, Onishi H, Tani M, Kinoshita H, Ueno M, Yamaue H. Indication and procedure for treatment of hepatolithiasis. Arch Surg. 2002;137(2):149-153.

15. Tabrizian P, Jibara G, Shrager B, Schwartz ME, Roayaie S. Hepatic resection for primary hepatolithiasis: a single-center Western experience. $J$ Am Coll Surg. 2012;215(5):622-626.

16. Shaw MJ, Dorsher PJ, Vennes JA. Cystic duct anatomy: an endoscopic perspective. Am J Gastroenterol. 1993;88(12):2102-2106.
International Medical Case Reports Journal

\section{Publish your work in this journal}

The International Medical Case Reports Journal is an international, peer-reviewed open-access journal publishing original case reports from all medical specialties. Previously unpublished medical posters are also accepted relating to any area of clinical or preclinical science. Submissions should not normally exceed 2,000 words or
Submit your manuscript here: https://www.dovepress.com/international-medical-case-reports-journal-journal

\section{Dovepress}

4 published pages including figures, diagrams and references. The manuscript management system is completely online and includes a very quick and fair peer-review system, which is all easy to use. Visit $\mathrm{http}: / / \mathrm{www}$. dovepress.com/testimonials.php to read real quotes from published authors. 